\title{
Neurodidáctica y autorregulación del aprendizaje, un camino de la teoría a la práctica
}

\author{
Álvaro Federico Muchiut; Rocío Beatriz Zapata; Alejandra Comba; Martín Mari; \\ Noelia Torres; Jéssica Pellizardi; Ana Paula Segovia *
}

Resumen. La autorregulación del aprendizaje apunta a generar un proceso autónomo en los alumnos; en base a este enfoque, consideramos que la tarea educativa debe dirigirse a la formación de competencias que permiten el aprender a aprender, logrando así un aprendizaje constante y continuo durante toda la vida en las diferentes situaciones y ámbitos en los que deba desenvolverse $y$, fundamentalmente, habilitar espacios en los que el estudiante genere nuevas formas de pensamiento y comportamiento. Por todo esto, comenzamos a investigar y buscar alternativas y estrategias variadas para poder modificar la forma en la que cada docente acerca los saberes a los alumnos, incidiendo en la metodología y estrategias de enseñanza, buscando que los alumnos se involucren en su propio aprendizaje, se responsabilicen por el mismo y sean conscientes de las modificaciones que se produce en ellos. Para abordar esta situación, desde la neurodidáctica creamos proyectos en distintos espacios curriculares, donde los profesores propiciaron que el aprendizaje esté centrado en el estudiante, considerándolo como eje del proceso, reconociendo, aceptando y respetando que es él quien realiza una construcción activa del conocimiento con la guía del profesor y sus compañeros. A lo largo de este escrito presentamos la articulación desde la teoría a la práctica a través de los diferentes espacios curriculares.

Palabras clave: neurodidáctica; autorregulación del aprendizaje; nivel secundario; aprendizaje autónomo.

\section{NEURODIDÁTICA E AUTORREGULAÇÃO DA APRENDIZAGEM, UM CAMINHO DA} TEORIA À PRÁTICA

Resumo. A autorregulação da aprendizagem visa gerar um processo autônomo nos alunos. Com base nesta abordagem, acreditamos que a tarefa educativa deve ser direcionada para a formação de competências que permitam aprender a aprender, alcançando assim uma aprendizagem constante e contínua ao longo da vida em diferentes situações e contextos que se apresentem e basicamente, proporcionando espaços nos quais o estudante gere novas formas de pensamento e comportamento. Por tudo isto, começamos a investigar e a procurar alternativas e estratégias variadas para mudar a maneira pela qual cada professor transmite o conhecimento para os alunos, com foco na metodologia e em estratégias de ensino, proArgentina.

*Fundación Centro de estudios Cognitivos, Instituto Superior de Neuropsicología, 
curando que os alunos se envolvam em sua própria aprendizagem, sejam responsáveis por ela e estejam cientes das mudanças que ocorram neles mesmos. Para lidar com esta situação, a partir da neurodidática criamos projetos em diferentes espaços curriculares, onde os professores consideraram que a aprendizagem esteja centrada no estudante, considerando-o como o núcleo do processo, reconhecendo, aceitando e respeitando que é ele quem realiza uma construção ativa do conhecimento, com a orientação do professor e de seus colegas de classe. Ao longo deste artigo apresentamos a articulação da teoria à prática mediante os diferentes espaços curriculares.

Keywords: Neurodidactics; Self-regulation of Learning; Secondary Level; Autonomous Learning.

\section{NEURODYDACTIC AND SELF-REGULATION OF LEARNING, A PATH FROM THEORY TO PRACTICE}

Abstract. The self-regulation of learning aims to generate an autonomous process in the students; Based on this approach, we consider that the educational task should be directed to the formation of competences that allow learning to learn, thus achieving a constant and continuous learning throughout life in the different situations and areas in which it must develop and, fundamentally, , enable spaces in which the student generates new ways of thinking and behavior. For all this, we began to investigate and look for alternatives and varied strategies to be able to modify the way in which each teacher brings knowledge to the students, focusing on the methodology and teaching strategies, looking for students to get involved in their own learning, Be responsible for it and be aware of the changes that occur in them. To address this situation, from the neurodidactics we create projects in different curricular spaces, where the teachers propitiated that the learning is centered on the student, considering it as the axis of the process, recognizing, accepting and respecting that it is he who realizes an active construction of knowledge with the guide of the teacher and his classmates. Throughout this paper we present the articulation from theory to practice through the different curricular spaces.

Keywords: Neurodidactics; Self-regulation of Learning; Secondary Level; Autonomous Learning.

\section{INTRODUCCIÓN}

La sociedad actual se encuentra en constante cambio, por lo tanto, los sujetos que asisten a las Instituciones Educativas en todos sus niveles se modifican año a año, existen transformaciones a nivel familiar, cultural, de pensamiento, a nivel personal, sentimental, de creencias, etc. A raíz de esto, la escuela debe prepararse para poder contener y dar respuesta a las demandas de estos sujetos cuya identidad se encuentra en construcción, posibilitando el aprendizaje en todas las áreas de su vida, no solamente en los espacios curriculares que rigen en el diseño curricular nacional y provincial, sino también otorgando la posibilidad de construir herramientas que le 
permitan desenvolverse en lo cotidiano y aprender en todos los ambientes posibles, para que el aprendizaje se lleve a cabo durante toda la vida y no únicamente en los años de escolarización.

Hoy, encontramos en las aulas del nivel secundario a adolescentes con pocas o nulas ganas, interés, motivación e iniciativa respecto al aprendizaje, por lo que consideramos menester realizar distintas intervenciones que modifiquen la situación, buscando revertir la actual actitud en los sujetos.

La Ley de Educación Nacional (2006), define en su artículo 29 que: “La Educación Secundaria es obligatoria y constituye una unidad pedagógica y organizativa destinada a los/as adolescentes y jóvenes que hayan cumplido con el nivel de Educación Primaria" y tiene como fin "habilitar a los/las adolescentes y jóvenes para el ejercicio pleno de la ciudadanía, para el trabajo y para la continuación de estudios" (art. 30). En cuanto a su organización, refiere que "se divide en dos ciclos: un Ciclo Básico, de carácter común a todas las orientaciones y un Ciclo Orientado, de carácter diversificado según distintas áreas del conocimiento, del mundo social y del trabajo" (art. 31).

En el último informe estadístico nacional del sistema educativo muestra que los niveles de promoción han mejorado y casi se han equiparado entre ambos ciclos, y que la problemática de ambos ciclos difiere: mientras que en el Básico el principal problema lo constituye la repitencia, en el ciclo orientado cobra mayor notoriedad la alta tasa de abandono. La repitencia y el abandono acumulado año a año redundan en una baja tasa de egreso para la educación secundaria. La mejora de estos indicadores en los últimos años se tradujo en un incremento de la tasa de egreso que en el período 2006-2013 ha aumentado casi un $21 \%$, (de 37,6\% en 2006 a un $45,4 \%$ en 2013), con un salto notable de 2008 a 2009. De la totalidad de alumnos en el ciclo orientado, en 2006 la mitad de ellos lograban graduarse, mientras que para 2016 el porcentaje se había elevado al 59,4\% (Ministerio de Educación y Deportes, 2017).

Estas estadísticas nos muestran la perentoriedad de un cambio en las prácticas pedagógicas cotidianas, con un índice de deserción tan alto, es menester rebatir dicha situación y generar cambios dentro del sistema.

Como centro educativo nos posicionamos desde la neurodidáctica, la cual es una rama de la pedagogía basada en las neurociencias que otorga una nueva orientación a la educación. Es la unión de las ciencias cognitivas y las neurociencias con la educación, que tiene como objetivo diseñar estrategias didácticas y metodológicas más eficientes, que no solo aseguren un 
marco teórico y filosófico, sino que promuevan un mayor desarrollo cerebral, (mayor aprendizaje) en términos que los educadores puedan interpretar (Paniagua, 2013).

En este sentido, las neurociencias tienen mucho que aportar a la didáctica del aprendizaje, en cuanto que es la ciencia que se encarga de estudiar el sistema nervioso central desde su funcionamiento neuronal hasta el comportamiento, cuyo propósito principal es entender cómo el encéfalo hace conducta. De ello podemos comprender que puede hacer numerosos aportes al proceso de enseñanza-aprendizaje, dado que hay cuatro mecanismos básicos que el cerebro utiliza para poder aprender: la motivación, la atención, la emoción y la memoria, mecanismos estudiados en profundidad por las neurociencias y que aportan un sustento teórico a los que trabajan en educación de cómo aprende el cerebro.

Conforme a esto, se ha implementado este enfoque de la neurodidáctica, estableciéndose como eje institucional el desarrollo de la autorregulación en los alumnos. Rosário y Poydoro (2012 citado en Fuentes y Rosário, 2013) exponen que el proceso de autorregulación del aprendizaje apunta al desarrollo del aprendizaje autónomo por parte del estudiante. Este proceso permite competencias como la autogestión y proactividad, autoconocimiento, responsabilidad y autocontrol del proceso de aprendizaje por parte del propio alumno.

Es el estudiante quien debe llevar a cabo una serie de acciones que favorezcan su desarrollo; como ser:

- Tomar la iniciativa en su proceso de aprendizaje.

- Llevar a cabo un diagnóstico previo de las necesidades propias de su aprendizaje, con o sin ayuda de otros.

- Formular metas de aprendizaje propias.

- Identificar los recursos humanos y materiales necesarios para alcanzar las metas de aprendizaje establecidas.

- Elegir e implementar las estrategias de aprendizaje adecuadas.

- Llevar a cabo un proceso de autoevaluación de los resultados del aprendizaje.

Por muchos años, el aprendizaje fue concebido como una actividad donde el sujeto que aprende se encontraba pasivo, receptivo y expectante a lo que el docente, considerado fuente de conocimiento, podía impartirle. Esta concepción de aprendizaje fue modificándose, y hoy en día es sabido 
que el aprendizaje es un proceso activo, que requiere de las capacidades y habilidades del sujeto que aprende, por lo que al considerar al aprendizaje autorregulado, debemos comprender que el mismo requiere de actividades del alumno que posibiliten el aprender. Normalmente los alumnos que autorregulan su aprendizaje monitorizan su comportamiento en relación con sus objetivos y reflexionan sobre los avances que se van produciendo, esto lleva a la satisfacción personal y aumenta su motivación para continuar mejorando su método de aprendizaje, lo que termina repercutiendo en buenos resultados académicos (Núñez, J. et al., 2006).

Teniendo en cuenta un estudio de revisión bibliográfica realizado por Rosario, P., et al (2014) aseguramos que el aprendizaje autorregulado fue motivo de investigación para distintos autores desde distintas teorías, pero independientemente de esto, existen algunas coincidencias que son necesarias para lograr un entendimiento profundo del concepto. Todos los investigadores defienden que el alumno a través de diferentes procesos puede regular activamente su cognición, motivación y comportamiento, con el fin de alcanzar determinados objetivos, mejorando a su vez su rendimiento académico. Por otro lado, se destaca la importancia del componente motivacional en el aprendizaje autorregulado; ya que el mismo requiere de esfuerzo, persistencia, tiempo para realizar las tareas, entre otras cosas, por lo que es necesario atender a esta dimensión para poder analizar y desarrollar la autorregulación. En relación a esto, en otra de sus investigaciones Zimmerman (2001), asegura que la autorregulación es un proceso complejo, donde confluye la metacognición, ya que implica la autoeficacia y actividad por parte del sujeto, por lo cual también incluyen procesos motivacionales y comportamentales para poder Ilevar adelante dicho proceso.

Printich (1998, citado en Rojas, 2008) elabora un marco teórico donde describe, conceptualiza y brinda distintas formas de intervenir en el aprendizaje autorregulado. El autor postula que para un buen rendimiento académico, no solo se debe hacer foco en las cuestiones cognitivas, sino que existen distintos factores que influyen para llegar al buen rendimiento. Estos factores son: la cognición, la motivación, el comportamiento y el contexto. Estos elementos influyen en el aprendizaje, y por ende en el rendimiento académico; por esto, es necesario realizar intervenciones que incentiven el desarrollo y funcionamiento armónico de los elementos mencionados.

El autor describe un modelo planteado desde cuatro fases, que permiten el desarrollo de la autorregulación en el aprendizaje:

- Fase 1: previsión, planificación y activación: conlleva la programación, el establecimiento de metas de aprendizaje, implica el 
conocimiento personal, el contexto y los conocimientos previos al realizar una actividad académica.

- Fase 2: monitoreo: se activa la mayor conciencia metacognitiva, posibilitando el seguimiento de los aspectos personales, de la tarea y del contexto que inciden en el rendimiento.

- Fase 3: control: se llevan a cabo diversos procesos de control y regulación sobre sí mismo, el contexto y la actividad.

- Fase 4: reflexión y redacción: el sujeto se autoevalúa y evalúa el contexto y la tarea, para luego determinar si requiere realizar modificaciones en conductas que no favorecen el proceso de aprendizaje.

En concordancia con Fuentes y Rosário (2013) consideramos que el aprendizaje debe resaltar su carácter significativo, permanente y sobre todo autónomo, destacando aquellas competencias y capacidades que permitan conseguir un aprendizaje autónomo y autorregulado. Es por ello que en este proyecto institucional se tuvo como fin principal la puesta en práctica del enfoque basado en la Autorregulación de los procesos de aprendizaje, con una metodología basada en la neurodidáctica, integrando el conocimiento de los procesos cerebrales implicados en el aprendizaje para una mejor enseñanza.

\section{MÉTODOS Y MATERIALES}

Esta investigación se realizó junto con profesores del nivel secundario, quienes construyeron y llevaron a la práctica proyectos donde por medio de las actividades brindadas y la metodología de enseñanza se pueda aproximar al desarrollo del aprendizaje autorregulado como fin último.

Dichos proyectos se llevaron a cabo con alumnos de $1^{\circ}$ y $2^{\circ}$ año del nivel medio (13 y 14 años), en cinco espacios curriculares: lengua, geografía, inglés, matemática y música, las últimas realizaron un trabajo en conjunto.

En el área de LENGUA, la propuesta fue a través de siete clases donde el tema central fue el cuento policial.

El objetivo fue que los alumnos conozcan el concepto de cuento policial; reconozcan su estructura; identifiquen la perspectiva antitética; distingan entre cuento policial de enigma y cuento policial negro 
Las siete clases se describen a continuación:

- Clase 1: conceptualización y explicación del cuento policial. Se realizó la lectura en pareja del cuento "La pieza ausente" de Pablo de Santis.

- Clase 2: estructura del cuento, lectura y puesta en común del libro "Corazón delator" de Edgar Allan Poe, identificando la estructura del mismo.

- Clase 3: clasificación del cuento enigma y cuento negro, se realizo un cuadro comparativo.

- Clase 4: lectura en pareja de "El vampiro de Sussex", confrontación antitética.

- Clase 5: características del cuento policial negro. Lectura en pareja de fragmentos de "Luna caliente" de Mempo Giardinelli, reconocimiento de las características; puesta en común.

- Clase 6: se realizo un trabajo practico; lectura del texto "Tres portugueses bajo un paraguas" de Rodolfo Walsh, identificar el tipo de cuento del que se trata, reconocer la estructura, caracterizar los personajes, y distinguir si pertenece al cuento policial de enigma o policial negro.

- Clase 7: se entregan las grillas de autoevaluación y coevaluacion.

A través de una secuencia de actividades se puso en acción las distintas fases de la autorregulación.

En primer término se puso en práctica lo que Zimmerman y Martinez-Pons (1990, citados en Núñez, J. et al, 2006) caracterizan como fase previa, que consta de brindarles a los estudiantes los objetivos esperados y las estrategias que serán empleadas. La literatura describe que los alumnos con objetivos orientados al aprendizaje están más centrados en el progreso de su aprendizaje que en la competición con sus iguales, y tienden a aprender más eficazmente que los alumnos con objetivos centrados en la realización.

Luego se puso en práctica la fase dos a través de distintas actividades enfocadas en procesos de autoinstrucciones que los ayuden a regular su aprendizaje. La investigación sugiere que las autoinstrucciones mejoran el aprendizaje de los alumnos, ya que la vocalización de los protocolos (e.g. algoritmos, fórmulas químicas) contribuye a la disminución de los errores cometidos. En esta instancia realizaron un proceso de autoobservación que les permitió observar debilidades y fortalezas. 
En la fase tres fue el momento de reflexión y de autoevaluación de los resultados obtenidos, los cuales se cotejaron con los objetivos iniciales, de manera de comprobar qué aprendizajes se produjeron y cuáles son aqueIlos puntos en los que debería mejorar. La auto-evaluación de los resultados escolares es normalmente uno de los procesos autoreflexivos iniciales, que implica la comparación de la información monitorizada con algún objetivo educativo concreto.

A su vez realizaron una coevaluación de su compañero de trabajo, basándose en el desempeño de su par a través de la observación de las distintas clases y resultados obtenidos, de modo de retroalimentar el aprendizaje autorregulado de su compañero y viceversa.

Para finalizar se realizó una heteroevaluación de doble entrada, por un lado la docente evaluó el proceso a nivel global del estudiante y de la misma manera los estudiantes observaron y evaluaron el acompañamiento docente.

A través de la valoración de los aprendizajes propios (autoevaluación), la valoración de un par (coevaluación) y la valoración por parte de un docente (heteroevaluación) se produce una retroalimentación permanente que propicia el aprendizaje autorregulado.

De este modo se tuvo en cuenta en la planificación y secuenciación didáctica del docente, las funciones ejecutivas de planificación, toma de decisiones y flexibilidad cognitiva.

En el área de GEOGRAFíA, se planteó como objetivo que los alumnos conozcan y comprendan el campo disciplinar de la geografía y la aplicación de los saberes a la vida cotidiana; logren generar una apropiación significativa de los saberes; establezcan una postura crítica y reflexiva ante el mundo que los rodea; logren establecer mecanismos de aprendizaje autorregulado que les permita un desarrollo autónomo en sus prácticas de aprendizaje.

Este proyecto se llevó a cabo a través de un trabajo práctico evaluativo, donde los alumnos pusieron en juego lo trabajado acerca de la definición de geografía, los hechos y fenómenos geográficos tanto físicos, biológicos como sociales y los principios geográficos.

Los estudiantes realizaron en grupos, una investigación acerca de un hecho o fenómeno geográfico que sucede o sucedió en Argentina o en Chaco y basándose en los principios básicos geográficos los describieron; para finalizar el trabajo, cada alumno o grupo de alumnos presentó la investigación a través de una en exposición con lámina o power point. En esta presentación 
los alumnos mismos debían moderar al resto de la clase, poner orden, dar las actividades y evaluar según las producciones de sus compañeros con una nota numérica.

Durante el trabajo, los alumnos que observaban realizaron una coevaluación, es decir, que mientras un grupo presentaba su trabajo, los compañeros (observadores) lo evaluaban mediante una grilla donde plasmaban fortalezas y debilidades del grupo y asignaron una nota. Asimismo, los alumnos que exponían, al finalizar su trabajo realizaron una autoevaluación, es decir, que cada integrante del grupo completó una grilla donde exponía las fortalezas y debilidades de su propio grupo.

De este modo se tuvo en cuenta en la planificación y secuenciación didáctica del docente, las funciones ejecutivas de planificación, toma de decisiones e inhibición.

En el área de MATEMÁTICAS y MúSICA el objetivo se centró en que el alumno planee su propio estudio; que pueda crear un hábito con respecto a la organización autónoma de sus propias actividades, que pueda organizarse al momento de estudiar, con respecto al material de estudio, tiempo de estudio, formas de estudiar y lugar de estudio, que logre supervisar su propia ejecución, autoevaluar sus resultados y por último confirme o modifique su conducta.

Este proyecto se desarrolló teniendo en cuenta las tres fases del proceso de autorregulación del aprendizaje, es decir, planificación, realización y autoevaluación.

Para la fase de planificación, se implementó el uso de la agenda escolar o calendario, donde alumno dejaba por escrito fechas de exámenes, trabajos prácticos, jornadas, etc. se orientó al estudiante para la realización de actividades para el hogar o trabajos prácticos, a través de la intervención del docente con preguntas como ¿Qué deben traer para la próxima clase?, ¿Cuándo deben entregar el trabajo? ¿Qué actividades del libro deben hacer?, además se realizó una lista de temas para la evaluación, donde el docente junto con el alumno determinó los temas de mayor relevancia para la evaluación.

Para la fase de realización, se llevaron a cabo trabajos prácticos grupales, donde el docente cumple el rol de guía para la organización del trabajo mediando con las siguientes preguntas: ¿Cómo van a empezar? ¿Dónde harán el trabajo? ¿Qué conocimientos previos necesitan para realizarlo? Además se implementó el registro de los alumnos, se solicitó a los mismos un registro escrito de lo que aprendieron y sintieron en la clase para reflexionar a partir del mismo sobre los aprendizajes alcanzados y lo que debemos mejorar o potenciar en nuestra conducta; se habilitaron espacios para preguntas 
reflexivas, donde el docente guía podrá intervenir con preguntas como ¿Están cumpliendo lo que esperaban hacer? ¿Se distraen mucho? ¿El tiempo les alcanza? ¿Bajo qué condiciones logran concentrarse?

Para la fase de autoevaluación, se trabajó sobre la evaluación en sí, y al finalizar la misma el docente plasma los resultados correctos en el pizarrón para que cada alumno lo pueda revisar y corregir si tuvo algún error, así también los alumnos realizaron una producción escrita acerca de cómo se sintieron durante el proceso de evaluación. Además se implementó una coevaluacion, donde se les solicitó a los alumnos que al finalizar una actividad de evaluación, se intercambien sus exámenes y se corrijan unos a otros.

De este modo se tuvo en cuenta en la planificación y secuenciación didáctica del docente, las funciones ejecutivas de planificación, toma de decisiones y memoria de trabajo.

En el área de INGLÉS, el objetivo general fue realizar un folleto turístico con información de la ciudad, en relación a datos numéricos sobre la misma, lugares turísticos, historia y tradición. El proyecto se desarrolló a través de cuatro fases/etapas.

En la primer fase/etapa se planifica y organiza los pasos a seguir para obtener la información necesaria en base al objetivo planteado, mostrando una participación activa en el aprendizaje empleando estrategias cognitivas como la organización, elaboración y recuperación de información.

La segunda etapa constituyó básicamente la búsqueda activa de información por parte de los alumnos, desarrollando las competencias necesarias para la investigación. Los estudiantes buscaron información a través de datos aportados por profesores de otras materias, familiares, búsqueda por internet o en libros de historia de la ciudad. Realizaron además un listado de preguntas para entrevistas con familiares, docentes, para luego determinar los datos relevantes de cada entrevista, visitaron lugares turísticos, tomando fotos de cada lugar. Con el objetivo de capacitar al alumno para el aprendizaje autónomo y permanente, cada estudiante trabajó activamente en la búsqueda de información como así también en la elaboración de oraciones y textos en inglés; todas las actividades se realizaron con la guía del docente a cargo quien se encargó de monitorear cada acción ayudando a regular su propio aprendizaje.

En la tercera etapa, el objetivo propuesto fue realizar conexiones con palabras, expresiones y frases conocidas por los alumnos, con palabras y frases nuevas en inglés. 
En la cuarta etapa, el objetivo fue reflexionar sobre lo realizado en las etapas anteriores, de forma individual y grupal. Los alumnos emitieron su opinión sobre lo trabajado a través de un video, escribiendo y enviando sus audios al grupo de whatsapp que integran junto con la profesora. Además se eligieron las imágenes y datos relevantes para finalizar la creación del folleto. En esta última etapa, se trata de hacer significativa la información y construir conexiones entre la información dada por el material a aprender y el conocimiento previo del alumno.

De este modo se tuvo en cuenta en la planificación y secuenciación didáctica del docente, las funciones ejecutivas de planificación, toma de decisiones y memoria de trabajo.

\section{RESULTADOS}

Los resultados observados fueron alentadores en relación al objetivo propuesto. Si bien esto es un avance en la implementación en el desarrollo del aprendizaje autorregulado a través de la neurodidáctica, se observaron cambios significativos a nivel cualitativo en los estudiantes, entre los cuales se destaca la planificación de las tareas, la reflexión, la toma de decisiones, autoevaluación constante, así como también la evaluación hacia los compañeros.

En relación a la utilización de la agenda, la gran mayoría de alumnos se muestran reticentes a la misma, y si bien la utilizan para cuestiones escolares, se encuentra en proceso de adquirir el hábito y apropiarse de dicha herramienta.

Por otro lado se observa que los alumnos que pudieron incorporar esta herramienta como estrategia de organización, lograron realizar las actividades de manera autónoma utilizando la agenda como apoyo durante el horario de clase así como en el hogar. Esto tuvo como resultado un mayor desempeño académico, ya que los alumnos recuerdan evaluaciones, trabajos prácticos, materiales para cada clase, etc. Estos resultados fueron observados por el equipo docente de la Institución, y se constató a través de informes anteriores y posteriores a las estrategias áulicas implementadas.

Esta investigación sigue en curso, ya que los alumnos continúan en proceso de desarrollar un aprendizaje autónomo y como Institución se siguen implementando distintas estrategias y metodología de enseñanza para fomentar dicho desarrollo. 


\section{DISCUSIÓN}

Se considera que la autorregulación es una habilidad cognitiva que debe ser enseñada a cada sujeto, a cualquier edad. En este proceso de enseñanza y aprendizaje las instituciones educativas abarcan gran parte de la vida de los niños, adolescentes y adultos, por lo que resulta necesario fomentar y habilitar espacios donde los alumnos puedan desarrollar esta habilidad.

Una serie de estudios realizados por Dignath, Buettner y Langfeldt (2008), con niños del nivel primario, reveló la posibilidad de desarrollar la autorregulación desde edades tempranas.

Como pudimos observar en los resultados obtenidos, la autorregulación en el aprendizaje posibilita la autonomía del alumno, la reflexión y el aprendizaje continuo, adaptándose a las circunstancias que se presenten; debido a que el alumno se constituye como eje central del aprendizaje, se vuelve indispensable la motivación y predisposición del mismo para lograr dicho aprendizaje. Zimmerman (2001) asegura que una característica fundamental en los alumnos que regulan su propio aprendizaje es la participación activa en el proceso de enseñanza-aprendizaje, desde nivel cognitivo, conductual y motivacional; por eso es fundamental comenzar con dicha enseñanza de autorregulación desde los primeros años de vida. El objetivo principal por el cual en los últimos años se apunta a la adquisición de la autorregulación del aprendizaje es por su implicancia en toda la vida del sujeto, aun al finalizar la escolarización; ya que, como demuestra el estudio realizado por Simons, Dewitte y Lens (2004, citado en Sánchez, P. et al, 2011), aquellos estudiantes cuya conducta se regula internamente tienen mayor interés, confianza y persistencia; usan estrategias profundas de aprendizaje y obtienen mejores resultados en los exámenes que aquellos estudiantes que están regulados externamente.

Para que la educación alcance el objetivo central de una formación integral para cada sujeto en situación de aprendizaje independientemente del nivel de escolaridad que se encuentre cursando, es necesario que el alumno cuente con herramientas para poder construir y dirigir su propio aprendizaje, ajustando acciones y estrategias que posibiliten el acercamiento al objeto de estudio, para esto cada institución y grupo docente debe brindar estrategias que incluyan actividades de selección y organización de la información, repaso, revisión e integración de lo nuevo a lo ya dado, posibilitando la significación del trabajo y favoreciendo un ambiente propicio para un buen trabajo áulico. 
En este sentido, la neurodidáctica tiene un sustento teórico propicio y estrategias prácticas para poder desarrollar esta autonomía que tanto se busca en las instituciones educativas, nos brinda procesos pedagógicos innovadores que mejoran el trabajo en el aula y que puedan lograr una interacción creativa con los procesos de enseñanza-aprendizaje.

La importancia de este enfoque novedoso, radica en que las investigaciones que desde ella se están generado contribuyen a la comprensión del cómo se activa el sistema nervioso cuando alguien que estudia se acercar a nuevos objetos de conocimiento y qué procesos se generan en su cerebro, en sus emociones y en el sistema nervioso.

Considerando los avances de las neurociencias podemos afirmar que todo educador es modificador del cerebro, con posibilidades de cambiar la estructura, la composición química y la actividad eléctrica del cerebro. EI educador, desde el enfoque de la Neurodidáctica se convierte en modificador de la estructura cerebral, de la composición química del cerebro y de la actividad eléctrica cerebral (Paniagua, 2013).

En este sentido, con la comprensión de las funciones ejecutivas, la motivación, la memoria, las emociones y la atención en los procesos de aprendizaje, los docentes tienen mayores herramientas conceptuales y metodológicas para propiciar un aprendizaje significativo y aplicable a la vida de los estudiantes.

\section{CONCLUSION}

Luego de llevar adelante los proyectos mencionados anteriormente, determinamos la importancia de trabajar sobre el desarrollo de la Autorregulación de los procesos de aprendizaje en los alumnos, ya que se observó avances en relación a la reflexión, autoevaluación, metacognición. Como se mencionó anteriormente, la investigación continúa, ya que el cuerpo docente se continúa perfeccionando en estrategias áulicas desde la neurodidáctica, y los alumnos continúan en proceso de desarrollo de la autorregulación, en acciones concretas de los alumnos se evidencian cambios que perfilarían hacia un aprendizaje autónomo.

Asimismo, como Institución Educativa apuntamos a establecer este enfoque como base de los procesos de aprendizaje que se generen en todos los espacios educativos. 
Se puede demostrar el gran avance de las neurociencias $\mathrm{y}$, en especial, de la Neuroeducación a comprender la forma de aprender del ser humano. Bajo estos enfoques, ahora se tienen bases sólidas para lograr una mejor educación, sin embargo, aún quedan grandes pendientes por descubrir y el cerebro sigue siendo un misterio para la humanidad. (Linares, 2016)

\section{BIBLIOGRAFÍA}

Barca-Lozano, A., Almeida, L.S., Porto-Rioboo, A.M., Peralbo-Uzquiano, M., Brenlla-Blanco, J.C. (2012) Motivación escolar y rendimiento: impacto de metas académicas de estrategias de aprendizaje y autoeficacia. Anales de psicología. 28 (3), 848-859

Dignath, C.;Buettner, G. y Langfeldt, H. (2008) How can primary school students learn selfregulated learning strategies most effectively? A meta-analysis on self-regulation training programmes. Educational Research Review 3, 101-129.

Fernández, E., Bernardo, A., Suarez, N., Cerezo, R., Núñez, J.C., Rosario, P. (2013) Predicción del uso de estrategias de autorregulación en educación superior. Anales de psicología. 29 (3), 865-875.

Fuentes, S; Rosário, P. (2013) Aprendizaje Autónomo y Mediación Cognitiva: Mediar para la Autorregulación del Aprendizaje: Un desafío educativo para el siglo XXI. Instituto Internacional para el Desarrollo Cognitivo, Indesco. Facultad de Ciencias de la Educación. Universidad de Chile.

Lanz, M.Z. (2006) Aprendizaje autorregulado. Un estudio sobre estrategias de aprendizaje en escuela secundaria. (Tesis de maestría). Universidad Nacional del Comahue, Neuquén, Argentina.

Ley de Educación Nacional (2006). № 26280. Boletín Oficial de la República Argentina Nº 31.062, p.1, publicado el 28 de diciembre de 2006. Buenos Aires, Argentina.

Linares, G. (2016) Aprendizaje significativo y neurociencia: la conexión del siglo XXI. Revista Iberoamericana de Producción Académica y Gestión Educativa, 4.

Ministerio de Educación y Deportes. Presidencia de la Nación (2017). Informe estadístico del Sistema Educativo Nacional.

Núñez, J.; Solano, P.; González-Pienda, J.; Rosário, P. (2006) El aprendizaje autorregulado como medio y meta de la educación. Papeles del Psicólogo, 27(3), 139-146.

Paniagua, M. (2013) Neurodidáctica: una nueva forma de hacer educación. Fides et Ratio-Revista de Difusión cultural y científica de la Universidad La Salle en Bolivia, 6(6), 72-77. Recuperado de http://www.scielo.org.bo/scielo.php?script=sci_arttext\&pid=S2071081X2013000100009\&lng=es\&tIng=es.

Rosario, P., Lourenco, A., Paiva, M.O., Núñez, J.C., González-Pineda, J.A., Valle, A. (2012). Autoeficacia y utilidad percibida como condiciones necesarias para un aprendizaje académico autorregulado. Anales de psicología. 28 (1), 37-44.

Rosario, P., Pereira, A., Hogemann, J., Núñez, A. R., Figueiredo, M., Núñez, J. C., Fuentes, S., Gaeta, M.L. (2014). Autorregulación del aprendizaje: una revisión sistemática en revistas de la base SciELO. Universitas Psychologica, 13 (2), 781-798. 
Sánchez, P. A., Valdés, A. A., Gantús, M. \& Vales, J. (2011). Propiedades psicométricas de un instrumento para medir la disposición hacia el estudio. CPU-e, Revista de Investigación Educativa, 12. Recuperado de http://www.uv.mx/cpue/num12/inves/ Sanchez-Cuervo-propiedades.htm

Rojas, H. (2008) Aprendizaje Autorregulado, motivación y rendimiento Académico. Liberabit, $14,15-20$,

Whitebread, D., Basilio, M. (2012) Emergencia y desarrollo temprano de la autorregulación en niños preescolares. Profesorado revista de currículum y formación del profesorado. 16(1), 15-34.

Zimmerman, B. (2001) Becoming a Self-Regulated Learner. Theory into Practice, 41(2), 64-70. 